\title{
Do Eye Movements During Shape Discrimination Reveal an Underlying Geometric Structure?
}

\author{
Bradley R. Sturz ${ }^{*}$, Ty W. Boyer ${ }^{1}$, John F. Magnotti², and Kent D. Bodily ${ }^{1}$ \\ ${ }^{1}$ Georgia Southern University, Statesboro, Georgia, USA \\ ${ }^{2}$ Baylor College of Medicine, Houston, Texas, USA \\ *Corresponding author (Email: bradleysturz@ georgiasouthern.edu)
}

Citation - Sturz, B. R., Boyer, T. W., Magnotti, J. F, \& Bodily, K. D. (2017). Do eye movements during shape discrimination reveal an underlying geometric structure? Animal Behavior and Cognition, 4(3), $267-285$. https://doi.org/10.26451/abc.04.03.06.2017

\begin{abstract}
Using a psychophysical approach coupled with eye-tracking measures, we varied length and width of shape stimuli to determine the objective parameters that corresponded to subjective determination of square/rectangle judgments. Participants viewed a two-dimensional shape stimulus and made a two-alternative forced-choice whether it was a square or rectangle. Participants' gaze was tracked throughout the task to explore directed visual attention to the vertical and horizontal axes of space. Behavioral results provide threshold values for two-dimensional square/rectangle perception, and eye-tracking data indicated that participants directed attention to the major and minor principal axes. Results are consistent with the use of the major and minor principal axis of space for shape perception and may have theoretical and empirical implications for orientation via geometric cues.
\end{abstract}

Keywords - Geometrical processing, Shape perception, Spatial cognition

Historically, the incorporation of subjective spatial perception has been a fundamental component of research investigating insect navigation (Cheng, 2012; Collett \& Zeil, 1998). Specifically, visual "snapshots" and visual "panoramas" taken from the goal location are suggested to be stored in memory of the mobile organism, and these subjective views are essential in view-based matching accounts of spatial learning (see Pecchia \& Vallortigara, 2010). Despite the cross-species variability in applicability, ideas at the intersection of navigation, view-based matching, and subjective spatial perception have offered an invaluable opportunity for the development and refinement of existing theories of spatial cognition.

Within spatial cognition, orientation by means of the geometric properties of an enclosure has garnered substantial empirical and theoretical attention. Such attention appears to be based, at least in part, on the fact that orientation by geometric properties is ubiquitous within the animal kingdom. Most any mobile animal that has been trained to approach a specific object located in the corner of a rectangular enclosure will respond to both the trained corner and its $180^{\circ}$ rotational equivalent at abovechance levels when the specific object is removed (see Cheng, Huttenlocher, \& Newcombe, 2013; Cheng $\&$ Newcombe, 2005, for reviews). Of primary theoretical interest, responding to these two locations is consistent with the notion that participants extract geometric information about the environment during training. Such extraction of geometric information occurs despite it being neither sufficient nor necessary to locate the originally trained location. As a result, this rotational error phenomenon appears to have widespread theoretical importance regarding implications for theories of learning, memory, and cognition. 
One long-standing question within this line of research relates to the nature of the geometric information extracted from the environment (see Cheng et al., 2013, for a review). More specifically, debate continues concerning the shape parameters by which participants orient with respect to the environment (Kelly, Chiandetti, \& Vallortigara, 2011; Kelly, Durocher, Chiandetti, \& Vallortigara, 2011; Sturz \& Bodily, 2011b, see also Sutton, 2009). One proposal is that participants extract the major and minor principal axes of space, which pass through the centroid and approximate the length and width of the entire space, respectively (for a detailed mathematical and mechanical definition, see Cheng, 2005; see also Bodily, Eastman, \& Sturz, 2011; Cheng \& Gallistel, 2005; Sturz, Gurley, \& Bodily, 2011; left panel, Figure 1). Alternatively, participants may rely upon medial-axis strategies whereby they extract a trunk-and-branch system, similar to the skeleton of a shape (see Ambosta, Reichert, \& Kelly, 2012; Blum, 1967; Cheng, 2005; Kelly et al. 2011a, 2011b; Kelly \& Durocher, 2011; see right panel, Figure 1, c.f., Feldman \& Singh, 2006).

\section{Principal Axis}

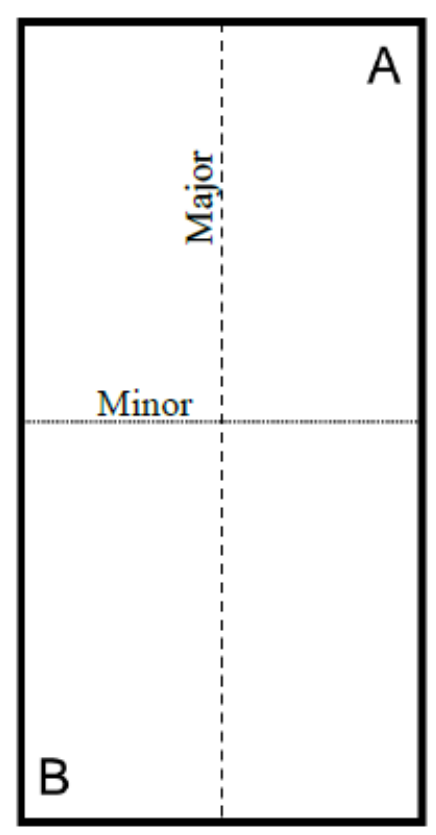

Medial Axis

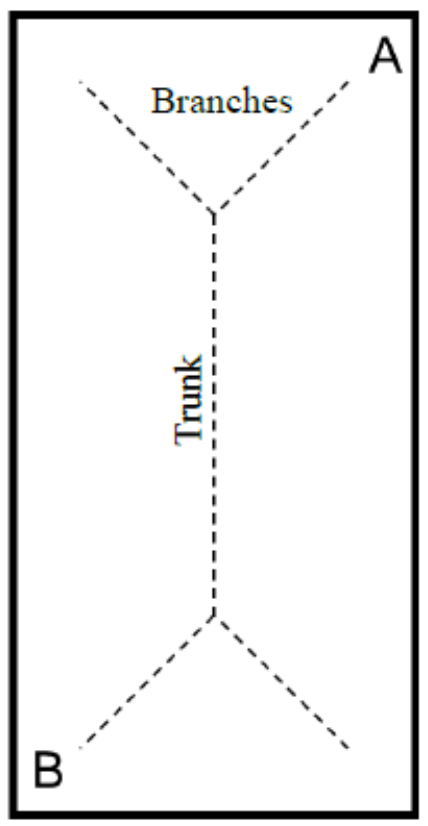

Figure 1. Illustration of principal-axis based (left panel) and medial-axis based (right panel) strategies. A represents trained location, and $\mathbf{B}$ represents the rotationally equivalent location.

Despite their geometrical independence, either principal or medial axis extraction can provide a theoretical explanation for responses in the reorientation task (for a review, see Cheng, 2005). The principal-axis approach suggests that when participants navigate to an object within the enclosure corner, they also incidentally learn where this is relative to the major principal axis of space (e.g., to the right) and/or the minor principal axis (e.g., to the left). In subsequent trials where the object is absent, searching relative to the major and minor principal axes (e.g., to the right and left, respectively) produces responses to both the correct and opposing corner. The medial-axis approach suggests that participants incidentally learn at which branch (e.g., right branch) at the end of the enclosure's central-trunk's terminal the corner resides. Subsequently, in the absence of the object, searching at the end of the correct and opposing trunk's terminal branches produces responses to the correct and opposing corners.

Although there is a rich history of incorporating subjective spatial perception into invertebrate navigation and orientation research (Stürzl, Cheung, Cheng, \& Zeil, 2008; Wystrach \& Beugnon, 2009; 
Wystrach \& Graham, 2012; for a review, see Cheng, 2012; Collett \& Zeil, 1998), the role of subjective spatial perception in other species has been underrepresented in the orientation literature. Such an underrepresentation appears difficult to reconcile with the theoretical issues at the forefront of the orientation literature. Specifically, the ongoing debate regarding the shape parameters by which participants orient with respect to the environment is largely predicated on an assumption that participants perceive an enclosure as it is objectively presented, and this assumption may undermine the ability to draw sound theoretical conclusions from orientation performance.

Two recent studies, however, have examined the effects of manipulating subjective spatial perceptual factors on orientation among human participants. Sturz, Kilday, and Bodily (2013) investigated the role of field of view (how much of an environment is visible from a single vantage point) in reorientation. Participants learned and then were tested in a reorientation paradigm, in either a small or large field of view condition. Those in the smaller field of view condition performed the reorientation task at chance levels whereas those in the larger field of view condition performed the reorientation task above chance levels. These results suggested that constraining field of view prevented extraction of geometric properties and spatial relations and resulted in an inability to use either global or local geometric cues for reorientation (see also Sturz, 2014).

Lee, Winkler-Rhoades, and Spelke (2012) also tested the effect of manipulating a subjective perceptual factor on orientation, by testing human children's ability to orient by environmental geometry in square enclosures. Two opposing walls in one of the square enclosures featured surface patterns that provided an illusion of depth, which was rationalized as creating a perceptible geometric cue (i.e., increased perceived length than width). As a result, it was predicted that the objectively square enclosure dimensions should subjectively appear rectangular. Consistent with this prediction, children were able to orient with respect to the environmental geometry despite its lack of objectively informative geometry.

The findings of these two studies underscore the importance of delineating the correspondence between objective shape parameters and subjective perceptions of space. Although orientation research generally necessitates the use of three-dimensional environments, we took a broader approach to examine the underlying assumptions of many spatial reorientation research paradigms - namely that the objective spatial structures presented to participants is isomorphic to the subjective spatial perception of such structures. Consistent with an insect navigation approach, we attempted to consider the importance of subjective perception in spatial tasks in an effort to inform and refine existing theories of spatial cognition.

To that end, the purpose of the current experiments was to exploit the opportunities that lie at the intersection of the subjective spatial perception, view-based matching, and spatial orientation lines of research. As a first attempt to inform the relationship between objective shape parameters and spatial perception, we used a psychophysical signal-detection approach, in which human participants viewed a simple two-dimensional shape stimulus and made a two-alternative forced-choice whether it was a square or rectangle. Importantly, we varied the length and width of the stimulus shapes to determine what objective parameters correspond to subjective determination of square versus rectangle judgments.

Specifically, we manipulated principal axis difference ratios (PADR) to determine the just noticeable difference (JND) for rectangles versus squares (see Sturz \& Bodily, 2011a). Sturz and Bodily proposed a hypothetical discriminability ratio between the major and minor principal axes of space, which they formalized as:

\section{Length of Major Principal Axis - Length of Minor Principal Axis Length of Major Principal Axis}

The PADR attempted to quantify the discriminability of the principal axis of space, and evidence suggested that the discriminability of the major relative to the minor principal axis influenced the usefulness of the axes as orienting cues.

Given that eye-tracking data has provided invaluable insights into the potential nature of perception and been used to classify underlying cognitive processes (see Henderson, 2017; Kardan, 
Berman, Yourganov, Schmidt, \& Henderson, 2015), participants' gaze was tracked throughout the task to further explore directed visual attention. The use of gaze data provided a unique opportunity to obtain evidence for, or against, the use of principal-axis or medial-axis based strategies. Specifically, the extent to which attention was directed to the vertical and horizontal axes of space versus the corners of the shapes should inform the use of the underlying decision strategy. More specifically, a principal axis strategy would suggest that participants should direct attention primarily to the major and minor principal axes. As a result, gaze data should be normally distributed around the central vertical and horizontal axes of the shape. In contrast, a medial axis strategy would suggest that participants should direct attention to both the primary vertical axis but also the corners. As a result, gaze data should be bimodally distributed across the shape in both the vertical and horizontal axes.

Although we acknowledge that two-dimensional shape perception differs in numerous ways from three-dimensional shape perception (and potentially its role in orientation), our goal is to outline a general research approach that integrates psychophysical analyses with eye-tracking data that could be applied across various environments. Importantly, the use of eye-tracking has also been used with nonhuman primates. For example, recent research has explored facial processing from a comparative perspective (Dahl, Wallraven, Bülthoff, \& Logothetis, 2009; Kano \& Tomonaga, 2010), and our general approach that integrates psychophysical analyses with eye-tracking data could be adapted for comparative purposes. As importantly, a similar forced choice task could be utilized across a wide variety of species and location of contact with the stimuli themselves may serve a similar role to eye-tracking data.

\section{Experimental Overview}

We attempted to determine how an objective measurement of shape corresponded to subjective perceptions of that shape by manipulating stimulus PADR values. On each trial, participants were presented with a single shape (either a square or a rectangle), and were asked to indicate via a key press whether it was a square or a non-square. By manipulating PADR values of the rectangles, we were able to discern what objective shape measurements mapped onto perceptions of square and perceptions of rectangle while simultaneously determining any individual differences in these perceptions.

\section{Method}

\section{Experiment 1A}

Participants. Eight males served as participants. Participants ranged in ages from $22-29$ years, had normal or corrected-to-normal vision, and provided consent for participating.

Apparatus. We constructed and implemented a computerized psychophysical 2D shape perception task. Stimuli appeared on the 512 x $285 \mathrm{~mm}$ flat-screen liquid crystal display (LCD) monitor of a Tobii TX-300 remote corneal reflection eye-tracking system, at 1,920 x 1,080 pixels resolution, with gaze recorded at $300 \mathrm{~Hz}$ (Tobii Technology Inc., Falls Church, VA, US). Participants produced manual responses by pressing the "c" (square response) and " $m$ " (not a square response) keys on a standard keyboard, with key to response mapping counterbalanced across participants. E-Prime (Psychology Software Tools, Inc., www.pstnet.com) software managed stimulus presentation, eye-tracking functionality, and response measurement.

Stimuli. The primary stimuli were black square and non-square rectangles presented on a screencentered 1,000 pixel diameter white circle that on average subtended $23.6^{\circ}$ visual angle horizontally, over a black background (see Figure 2). We created the stimuli by selecting five base size values: 100, 150, 200,250 , and 300 pixels, which on average subtended $2.4^{\circ}, 3.6^{\circ}, 4.8^{\circ}, 6.0^{\circ}$, and $7.2^{\circ}$, respectively. Squares, of course, involved applying the base size value to both the height and width dimensions. Nonsquare rectangles, by contrast, adopted the base value for either the height or width dimension, with the other dimension derived as a function of positive additions to the base value that produced a height:width principal axis difference ratio that assumed values 0.05 through 0.25 in increments of 0.05 (see Figure 3 ). 
The stimuli were presented in one of nine positions, formed by positively and negatively deviating horizontally and vertically by 0 or 32 pixels (i.e., $0.8^{\circ}$ spatial shifts) from screen center. Stimuli themselves were either oriented vertically $\left(0^{\circ}\right.$ along principal axis) or horizontally $\left(90^{\circ}\right.$ along principal axis). Thus, there were 45 unique square and 450 unique rectangle sizes $\mathrm{x}$ positions presented to participants. To compensate for the difference in probabilities of a square or rectangle occurring on a given trial, each unique square size $\mathrm{x}$ position was presented to each participant 10 times.

Procedure. First, we calibrated the eye-tracking system for each participant using a nine-point on-screen calibration routine. Participants then read an instructions page, which informed them that on each trial they would see a stimulus shape, that their task was to decide whether or not the shape was a square, and how to respond to the presented stimuli (see Appendix A). They then completed 10 training trials, all with accuracy and response time feedback. Participants saw the feedback for $1000 \mathrm{~ms}$ on the first training trial, but then this presentation time decreased incrementally over the course of the 10 training trials to $500 \mathrm{~ms}$. After training, participants began the experiment proper, and completed 18 blocks of 50 trials, all presented in fully random order, for a total of 900 trials. Each trial involved the following sequence: a fixation cross presented for $1000 \mathrm{~ms}$, followed by the square or rectangle stimulus for $900 \mathrm{~ms}$, followed by a blank response screen for $600 \mathrm{~ms}$, followed by feedback or a blank screen ISI for $500 \mathrm{~ms}$ (see Figure 2). Thus, each trial took three-seconds to complete. Feedback was provided on $40 \%$ of the trials; specifically, on all trials where the base size value was 150 or 250 pixels. Trials where participants provided no response were repeated.

\section{Experiment 1B}

To isolate the point of indifference and to illuminate the correspondence between objective presentation and subjective perception of shape, we needed to conduct a follow-up experiment using a finer resolution of PADR values.

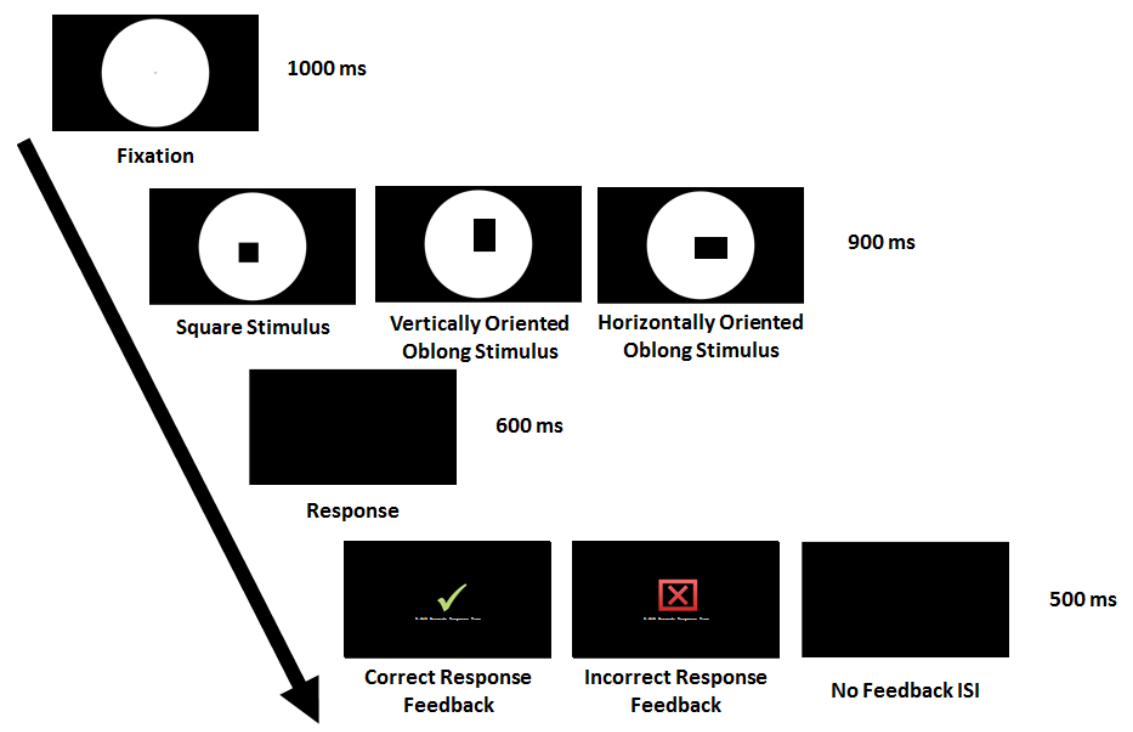

Figure 2. Trial structure of the present task. 


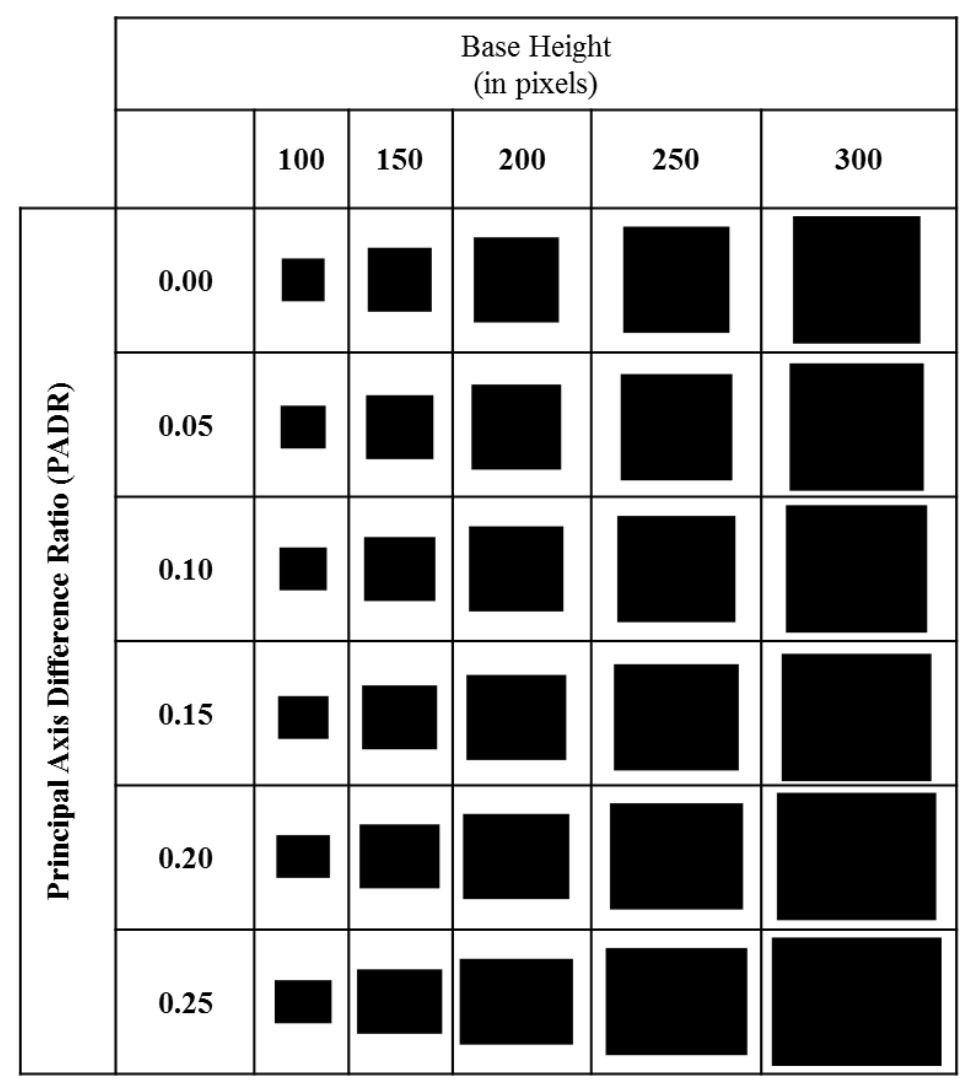

Figure 3. Stimuli used in Experiment 1A. Please note that stimuli are not to scale.

Participants. The same eight male students who participated in Experiment $1 \mathrm{~A}$ served as participants for Experiment 1B.

Apparatus, Stimuli, and Procedure. The apparatus, stimuli, and procedure were identical to Experiment 1A with the exception that we modified that the range of the values for the principal axis difference ratio such that stimuli assumed values of $0,0.025,0.05,0.06,0.07,0.08,0.09,0.1,0.125$, and 0.15 . In order to reduce the number of trials required, subsets of the base size values and positions within the circular space from Experiment 1A were used: 100, 200, and 300 pixels, which, on average, subtended $2.4^{\circ}, 4.8^{\circ}$, and $7.2^{\circ}$, respectively, and presentation within four positions, formed by positively and negatively deviating horizontally and vertically by 32 pixels (i.e., $0.8^{\circ}$ spatial shifts) from screen center. Thus, there were 12 unique square and 216 unique rectangle sizes x positions presented to participants. To compensate for the difference in probabilities of a square or rectangle occurring on a given trial, each unique square size $\mathrm{x}$ position was presented to each participant 18 times, resulting in a total of 432 trials per participant.

\section{Analysis Method}

Eye-Tracking. To analyze the eye-tracking data we divided each shape into 144 equally sized bins and computed the number of gaze samples that occurred within each bin for each shape and each participant. To illuminate where participants were attending during the task, we computed the proportion of gaze samples that fell within each bin relative to the $\mathrm{x}$-axis (i.e., vertical deviations from the primary horizontal axis of space) and the $y$-axis (i.e., horizontal deviations from the primary vertical axis) for each participant. 
Psychophysical models. To characterize individual differences in this task, we used a simple probabilistic model. The model assumes that, when shown a shape, the participant makes a noisy measurement of its principal and minor axes and calculates the PADR (equation 1). The participant then decides if this measured PADR is more consistent with a square (the true PADR is 0) or a rectangle (the true PADR is greater than 0 ). The observer takes into account the sensory noise in the system and does not simply respond "rectangle" whenever the measured PADR is greater than 0. Instead, the optimal observer relies on Bayes' rule to quantify the relative likelihood that the true shape is a Rectangle vs. a Square given the noisy PADR measure, $x$ :

$$
d=\frac{P(\text { Rect } \mid x)}{P(\text { Square } \mid x)}=\frac{P(x \mid \text { Rect }) \times P(\text { Rect })}{P(x \mid \text { Square }) \times P(\text { Square })}
$$

When $d$ is greater than 1 , the participant classifies the shape as a Rectangle. To ease calculation, we take the log of this ratio and compare $d$ to 0 , rather than 1. We assume unbiased Gaussian noise on the measurements and the decision rule simplifies to checking if the measured PADR falls outside a region near 0 . The size of this region is determined by the participant's prior belief about the likelihood of squares vs. rectangles and the amount of noise in the sensory system. We also modeled a guessing parameter to account for guessing and attentional lapses (effectively allowing "rectangle" responses to be $<1$ even for very large values of PADR). We found the best-fitting parameters for each participant by minimizing the squared error (we scale the error by the log of the number of observations, to give more weight to PADRs with more trials) between the predicted and actual data for each participant using a Nelder-Mead type algorithm implemented in R (R Core Team, 2014).

Although a good fit of our Bayesian model is a prerequisite for its endorsement, we cannot make strong conclusions without quantitatively comparing it to alternative models. To assess the relative suitability of our theoretically-motivated model, we compared its performance with two simpler models, and one more complex model. The simpler models were formed by fixing parameters in the current model to canonical values. The two-parameter, no-guessing model fixes the guessing parameter at 0.0 ; the oneparameter, noise-only model fixes the guessing parameter at 0.0 and fixes the priors at 0.5 (equal priors). For the more complex model, we adopted a curve-fitting approach based on a generalized logistic function:

$$
P(\text { rect })=L+\frac{U-L}{1+e^{-k\left(x-x_{0}\right)}}
$$

where $\mathrm{L}$ and $\mathrm{U}$ are the lower and upper asymptotes, respectively, $k$ controls the sensitivity to changes in PADR values, $\mathrm{x}_{0}$ is the midpoint of the function, and $x$ the empirical value of the PADR. Although not theoretically motivated, the increased number of parameters of the generalized logistic function allow for a more flexible response function. A better fit of this more complex model would suggest that the current model is deficient in fitting a particular aspect of the curve that could be addressed by augmenting the model in theoretically meaningful ways. For instance, the parameters $U$ and $L$ independently control the upper and lower asymptotes for responding "rectangle" at the highest and lowest levels of PADR. In contrast, the three-parameter Bayesian model enforces a complementary relationship between the upper and lower bounds (upper $=1-$ lower) at extreme PADR values.

To compare models, we use the Bayesian Information Criterion (BIC). BIC assesses the likelihood of the data given the model's parameters, with a penalty on free parameters that is proportional to the log of the number of trials. The absolute magnitude of BIC is not of interest, but the difference in BIC between models fit to the same data provides a measure of the relative log-likelihood of the data given each model. 


\section{Results}

The similarity of the two experiments allowed us to analyze the results together - analyzing them separately resulted in no qualitative difference in the results.

Behavioral responses. Across participants, as the PADR increased, the reports of rectangle also increased, although there were noticeable individual differences. Figure 4 shows that despite the differences in perception at each PADR, the simple model captures the data well (mean absolute error across participants $=4.5 \%$, range $1.5 \%$ to $7.7 \%$ ). The good fit of the model suggests that the 3-parameter model adequately characterizes performance across the 12 PADR values. Figure 5 shows the average reports of rectangle responses across the levels of PADR for each participant, along with the predicted values of the mean fitted model (generated from the average of the model parameters across participants).

The first parameter, the prior, functions as a threshold to control how large the measured PADR must be for the participant to reliably classify the shape as a rectangle. All participants had a value greater than 0 , ranging from 0.04 to $0.07(M=0.05)$. The sensory noise parameter controls how precisely the PADR is measured, controlling the sharpness of the square/rectangle boundary. One participant (S4) had very little noise (0.006), but, overall, the PADR was measured with considerable noise $(M=0.04, S D=$ 0.02 ), relative to the prior value. The guessing parameter (interpreted as the percentage of trials that were guesses) showed a strong contribution in two participants (values of $12 \%$ and $25 \%$ ), but on average was less than $10 \%$.

Eye-tracking results. Due to a calibration issue, we were unable to obtain useable eye-tracking data from one participant in Experiment 1A.

Horizontal deviations from the vertical axis of space. Figure 6 (left panel) shows the mean proportion of gaze sample deviations from the vertical axis of space. A one-way repeated measures ANOVA on proportion of gaze samples within each of the deviation bins ( -0.6 to $-0.5,-0.5$ to $-0.4,-0.4$ to $-0.3,-0.3$ to- $0.2,-0.2$ to $-0.1,-0.1$ to $0.0,0.0$ to $0.1,0.1$ to $0.2,0.2$ to $0.3,0.3$ to $0.4,0.4$ to $0.5,0.5$ to 0.6 ) revealed a main effect of deviation bin, $F(11,66)=9.08, p<0.001, \eta_{p}{ }^{2}=0.6$. Given 12 bins, the proportion of mean gaze samples falling into each bin by chance is 0.083 . The mean proportions of gaze samples falling into deviation bins -0.6 to $-0.5,-0.5$ to $-0.4,0.3$ to 0.40 .4 to 0.5 , and 0.5 to 0.6 were significantly less than would be expected by chance, $t \mathrm{~s}(6)>2.73 \mathrm{ps}<0.05$. The mean proportions of gaze samples falling into deviation bins -0.2 to $-0.1,-0.1$ to 0.0 , and 0.0 to 0.1 were significantly greater than would be expected by chance, $t \mathrm{~s}(6)>2.6, p \mathrm{~s}<0.05$. The mean proportions of gaze samples falling into the remaining deviations bins did not differ from chance, $t \mathrm{~s}(6)<2.4, p \mathrm{~s}>0.05$. Collectively, these analyses indicate that participants' visual attention tended to cluster near the primary vertical axis of space.

Vertical deviations from the primary axis of space. Figure 6 (right panel) shows the mean proportion of gaze sample deviations from the vertical axis of space. A one-way repeated measures ANOVA on proportion of gaze samples within each of the deviation bins ( -0.6 to $-0.5,-0.5$ to $-0.4,-0.4$ to $-0.3,-0.3$ to- $0.2,-0.2$ to $-0.1,-0.1$ to $0.0,0.0$ to $0.1,0.1$ to $0.2,0.2$ to $0.3,0.3$ to $0.4,0.4$ to $0.5,0.5$ to 0.6 ) revealed a main effect of deviation bin, $F(11,66)=2.08, p<0.05, \eta_{p}{ }^{2}=0.26$. Given 12 bins, the proportion of mean gaze samples falling into each bin by chance is 0.083 . The mean proportion of gaze samples falling into deviation bins 0.2 to -0.3 and 0.3 to 0.4 were significantly less than would be expected by chance, $t \mathrm{~s}(6)>2.6 \mathrm{ps}<0.05$. The mean proportion of gaze samples falling into deviation bins -0.2 to -0.1 was significantly greater than would be expected by chance, $t(6)>2.6, p s<0.05$. The mean proportion of gaze samples falling into the remaining deviations bins did not differ from chance, $t \mathrm{~s}(6)<2.2, p \mathrm{~s}>0.05$. Collectively, these analyses indicate that participants' visual attention tended to cluster near, but with a slight bias above, the primary horizontal axis of space. 
Sturz et al. 275
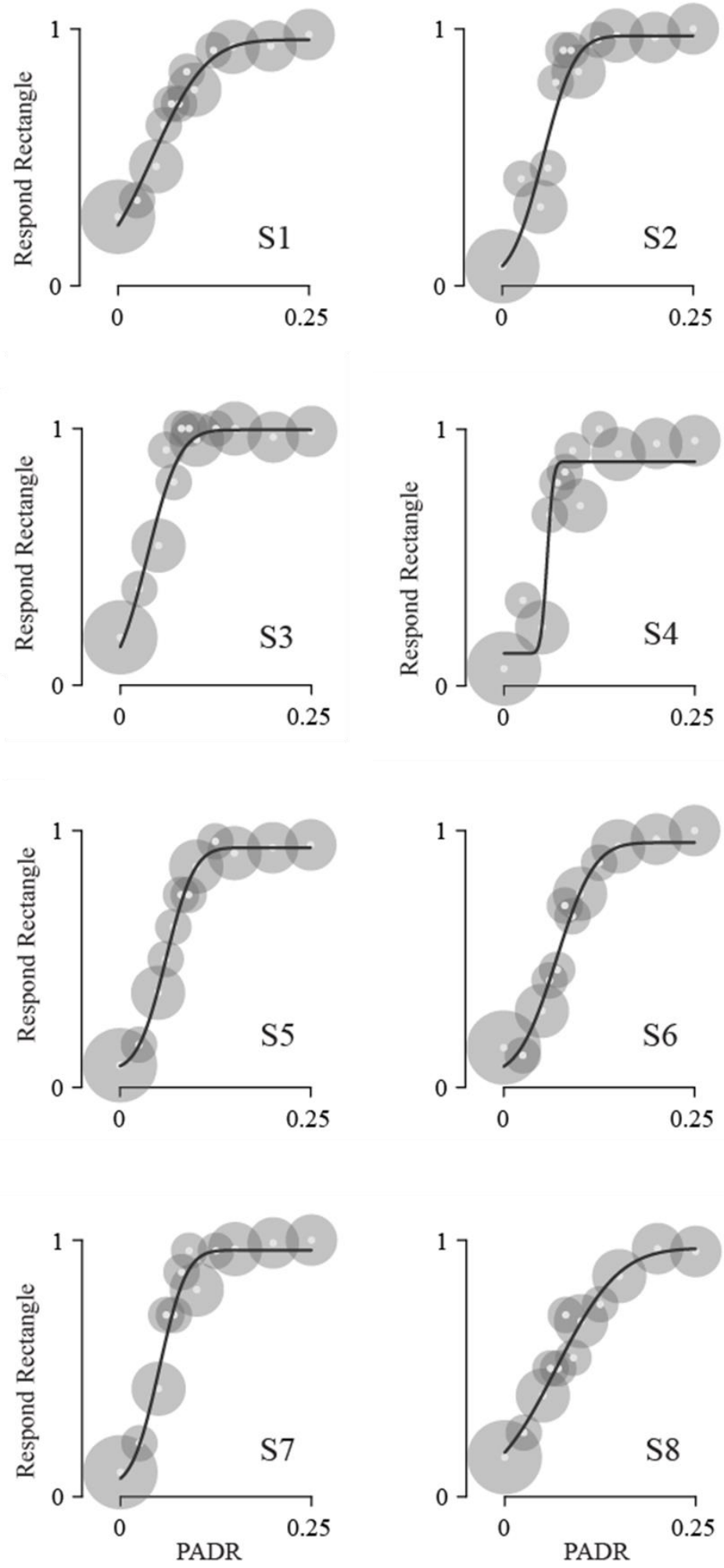

Figure 4. Mean proportion of rectangle responses plotted by Principal Axis Difference Ratio. Size of circle is proportional to the $\log$ of the number of trials. 


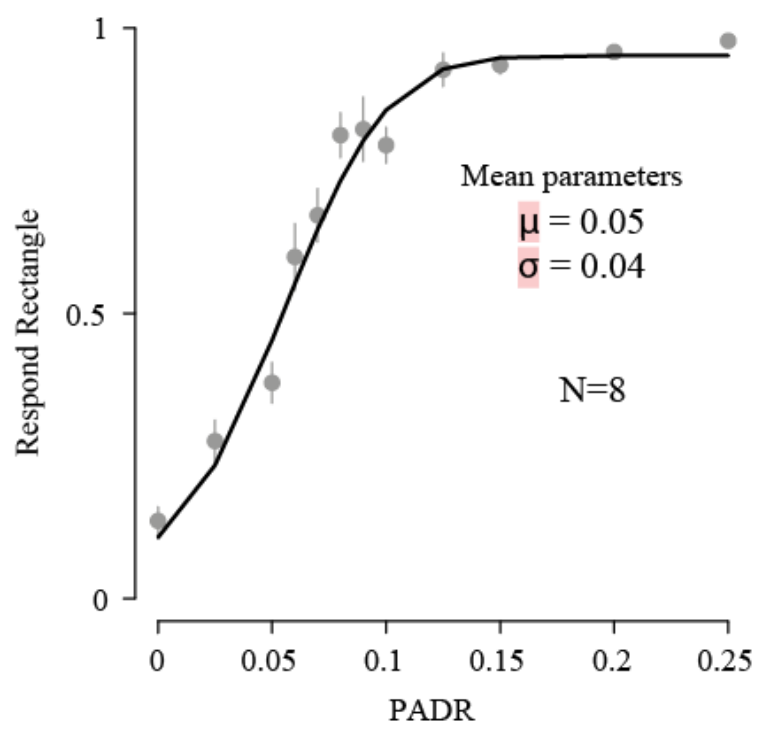

Figure 5. Average reports of rectangle responses across the levels of PADR for each subject, along with the predicted values of the mean fitted model (generated from the average of the model parameters across subjects). Error bars represent standard errors of the means.
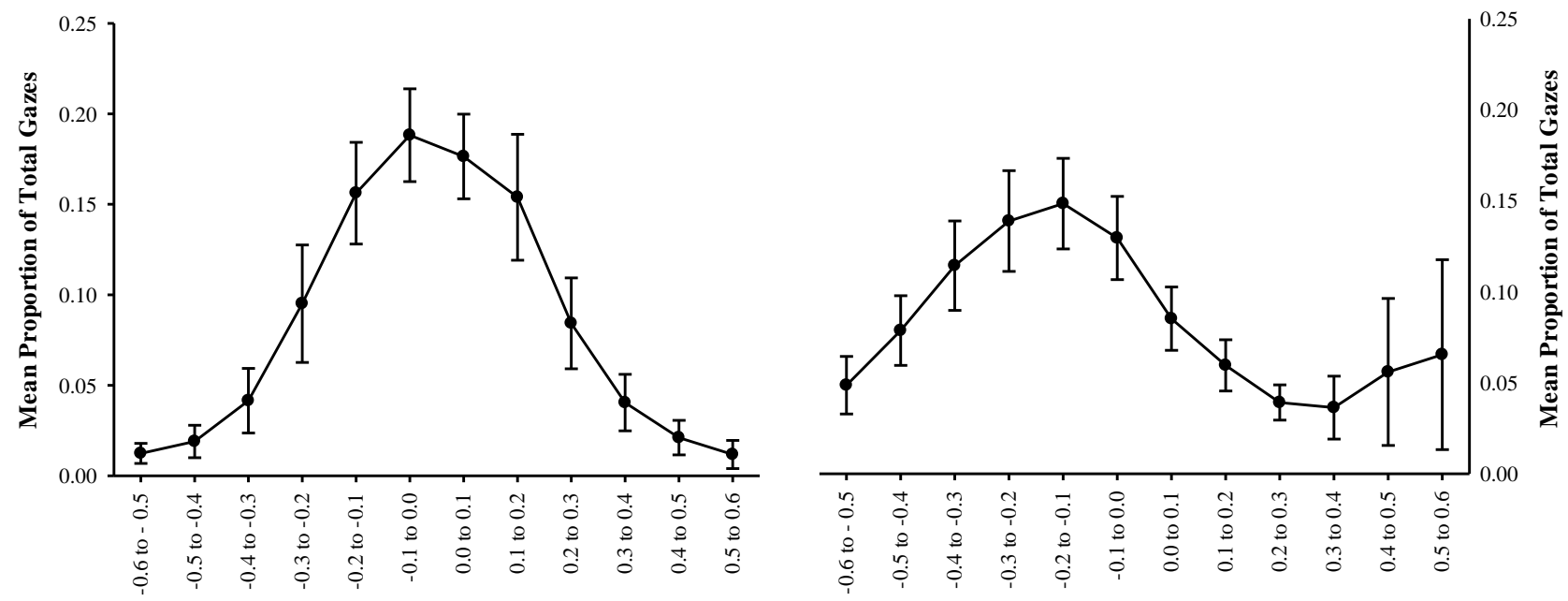

Horizontal Deviations from the Primary Vertical Axis of Space Vertical Deviations from the Primary Horizontal Axis of Space

Figure 6. Left panel. Mean proportion of gaze samples plotted by horizontal deviations from the primary vertical axis of space. Right panel. Mean proportion of gaze samples plotted by vertical deviations from the primary horizontal axis of space. Error bars represent standard errors of the means. Dashed lines represent chance performance (i.e., 0.083).

Deviations from principal and medial axes. We used a dispersion fixation detection algorithm that clustered gaze points within $1^{\circ}$ of visual angle of one another for a minimum series of $100 \mathrm{~ms}$ into discrete fixations. Figure 7 shows the location of fixations that fell within the boundaries of the stimuli for squares (top), vertically oriented rectangles (middle), and horizontally oriented rectangles (bottom) with the principal axes (left) and medial axes (right) overlaid on each stimulus type collapsed across PADR values, base heights, and base widths. To more directly test which potential global summary parameter better fit eye-tracking data, we also independently computed the distance of fixations from the principal 
and medial axis of each stimulus (for a similar approach, see Firestone \& Scholl, 2014). We analyzed all of the 6458 detected fixations that fell within the boundaries of the square and non-square stimuli. The mean deviation of fixations in pixels from the principal axes $(M=20.21,95 \%$ CI \pm 0.38$)$ was significantly less than that of the mean deviations of fixations that fell within the boundaries of the stimuli from the medial axes $(M=25.52 ; 95 \%$ CI \pm 0.49$)$, as confirmed by a paired samples $t$-test, $t(6457)=$ 16.63, $p<0.001, d=0.21$. In addition, 3781 (58.6\%) of all of the fixations were closer to the principal axes than the medial axis, and this was more than would be expected by chance, $\chi^{2}(1, N=6458)=$ $188.73, p<0.001$.
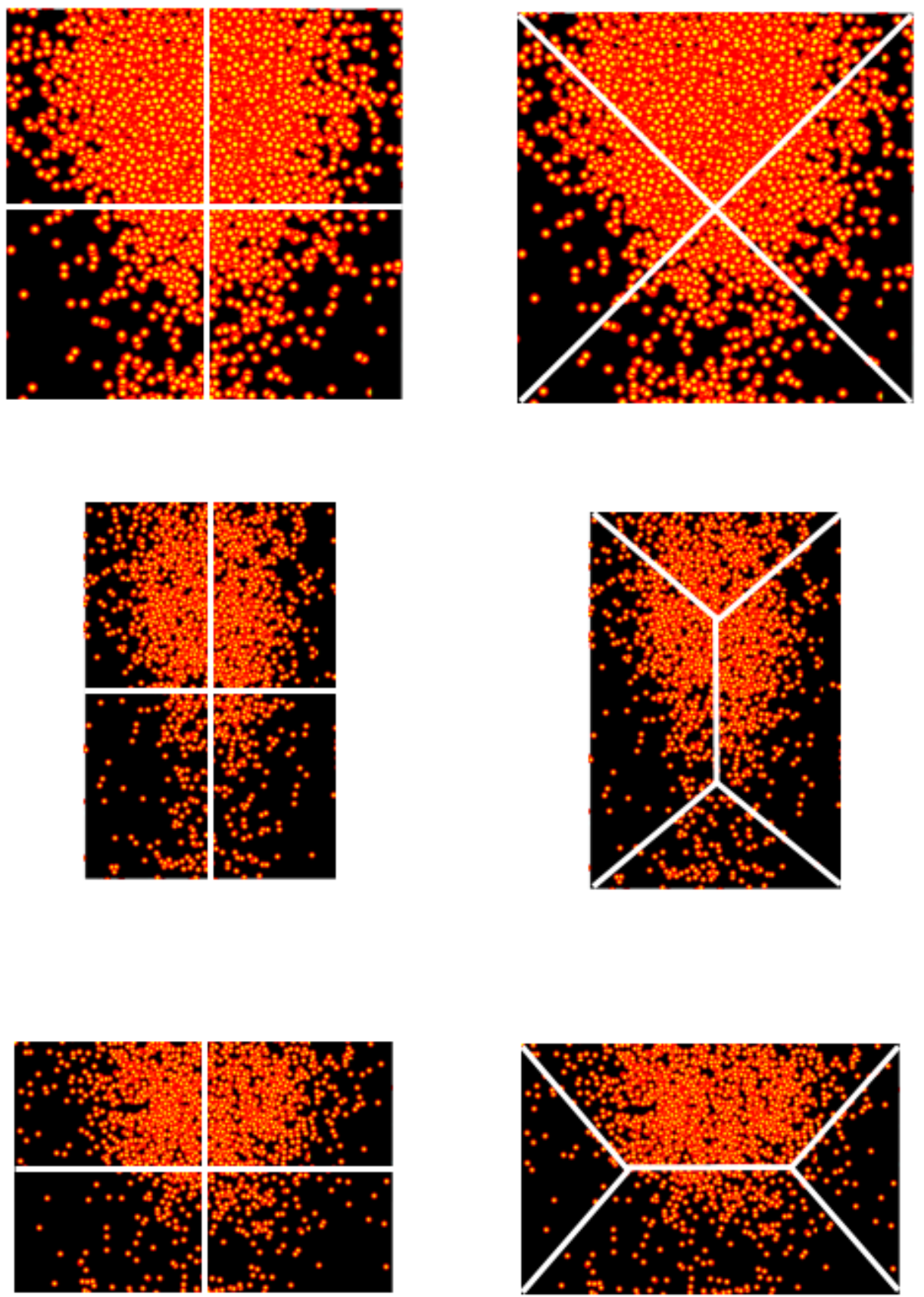

Figure 7. Fixations on squares (top), vertically oriented rectangles (middle), and horizontally aligned rectangles (bottom) with principal axes (left) and medial axes (right) overlaid on stimuli (represented by white lines). Please note that stimuli are not to scale and fixations were collapsed across PADR values, base heights, and base widths. 
Combined behavior and eye-tracking. Our final analysis compared the gaze deviations with the shape judgments directly. For each level of PADR, we calculated the proportion of rectangle reports and the mean horizontal and mean vertical gaze deviations (Figures 8 and 9, respectively). For each participant, we calculated the linear correlation between rectangle report and gaze deviation (both horizontal and vertical). Presumably, directed attention to the major and minor principal axes of space should result in a relationship that does not deviate significantly from 0 . In contrast, directed attention to the trunk and branches of a medial axis should result in a relationship that does deviate significantly from 0 .

We tested for a group-level relationship using a one-sample $t$-test (testing against 0 ) on each gaze deviation type. We found no consistent relationship between rectangle perception and horizontal deviations from the vertical axis $[M=-0.12,95 \% \mathrm{CI} \pm 0.27] ; t(7)=-0.9 p=0.4]$ nor between perception and vertical deviations from the horizontal axis $[M=0.8,95 \% \mathrm{CI} \pm 1.37] ; t(7)=1.2, p=0.3]$. Collectively, the eye-tracking data suggest that most participants were directing attention to the primary axes of space ( 0 deviation from $\mathrm{x}$ and 0 deviation from $\mathrm{y}$ ). The lack of relationship between eye-gazes and perception of rectangle/square at given PADR values suggests that differences in perception of rectangle/square is driven by an inability to differentiate length of $\mathrm{x}$ axis from length of $\mathrm{y}$ axis (i.e., major principal axis compared to minor principal axis). In short, these data support an interpretation that participants utilized the major and minor axes of space to determine decisions, and such an interpretation is aligned with what is suggested to drive orientation behavior.

Model comparisons. We assessed the adequacy of our three-parameter Bayesian model by comparing its fit (calculated using Bayesian Information Criterion, BIC; lower is better) with three alternative models. Two models, the no-guessing model and the noise-only model, were constructed by fixing free parameters in the current model. The third model consisted of a 4-parameter logistic function that, although atheoretical, provides a loose upper bound for the variability that might be explained by any S-shaped curve.

Across subjects, our proposed 3-parameter Bayesian model led to the lowest overall BIC (mean $\mathrm{BIC}=1041)$, the four-parameter logistic model second $(1046$; mean difference compared to 3-parameter model $=4.43, S D=3)$, followed by the no-guessing model $(1059$; mean difference $=17.7, S D=26)$ and the noise-only model (49847; mean difference $=48806, S D=10679)$. The high standard deviation for the BIC comparison of the two- and three-parameter models is indicative of individual differences in the model fits. Although the 3-parameter model was the best-fitting model for six of the eight subjects, the two-parameter, no-guessing model fit best for two of the eight subjects. The better fit of the no-guessing model for 2 subjects (S6 and S7 in Figure 4) is indicative of the low rates of putative guessing, as can be seen by lower (upper) response asymptotes near 0.0 (1.0). The overall superior fit of our three-parameter model suggests that it is capturing observed behavioral variability without overfitting.

\section{Discussion}

Results in the present two-dimensional shape discrimination task provide fundamental knowledge about the relationship between object shape parameters and perception of those shapes. Specifically, the present results suggest that rectangles with PADR values of 0.06 and below are perceived as squares. Importantly, the present results also provide a method in which to delineate this JND for individual participants. As importantly, the integration of eye-tracking data appears to provide results for the use of a principal-axis based strategy for shape determination as visual attention was directed mostly to the primary horizontal and vertical axes of space. This unimodal distribution in gaze data is not only consistent with the use of principal-axis based strategy but also inconsistent with a medial-axis based strategy. Specifically, the use of a medial axis strategy should result in gaze data being not only bimodally distributed but also systematically deviating away from the center of the stimuli and concentrated near the stimulus boundaries. In short, these data support an interpretation that participants are utilizing the major and minor axes of space to determine decisions, and such an interpretation is aligned with what is suggested to drive orientation behavior. 
Sturz et al. 279
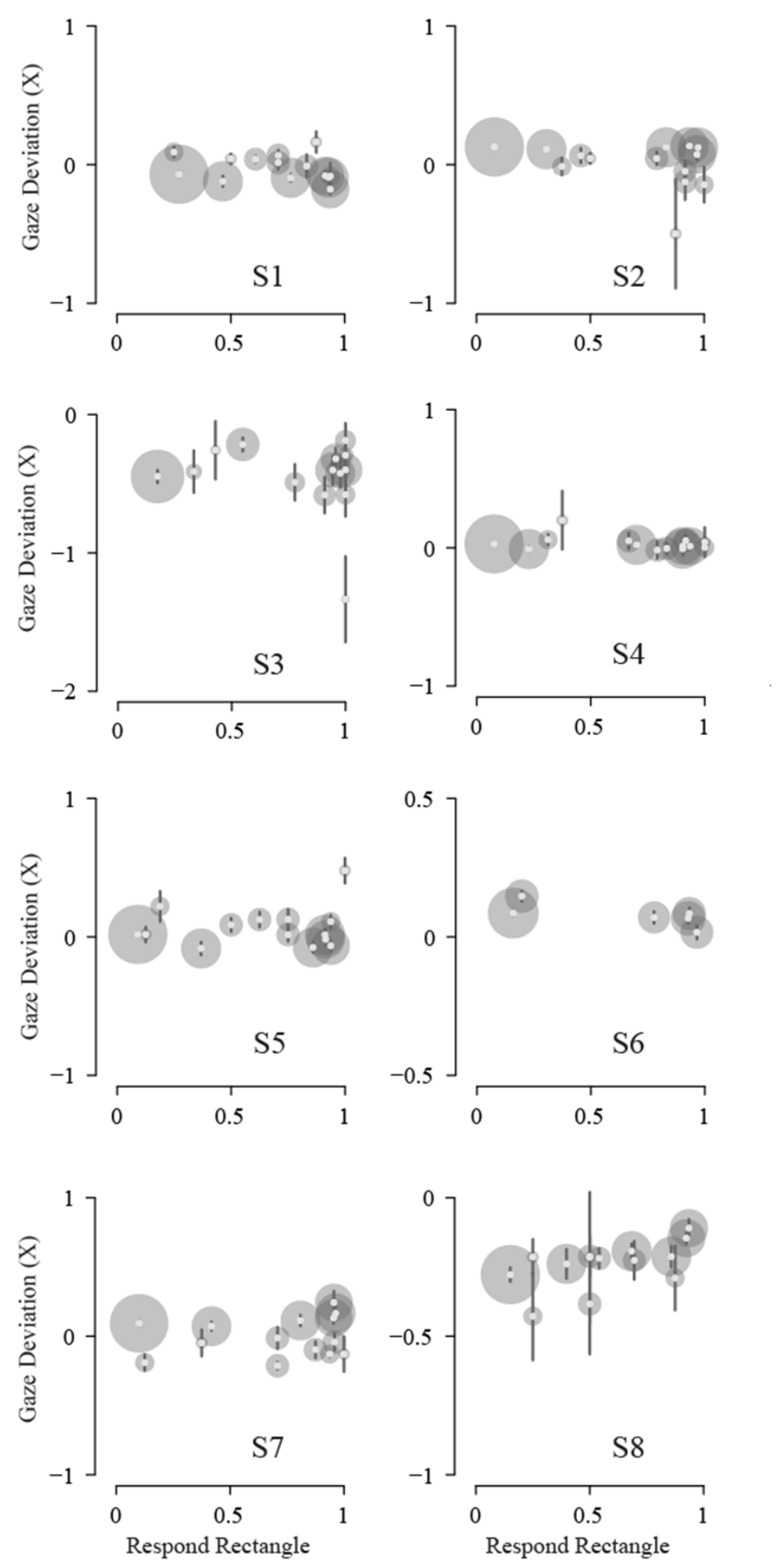

Figure 8. Gaze deviations plotted by proportion of rectangle responses for horizontal deviations from the primary vertical axis of space. Size of circle is proportional to the log of the number of trials. Error bars represent standard errors of the means. 

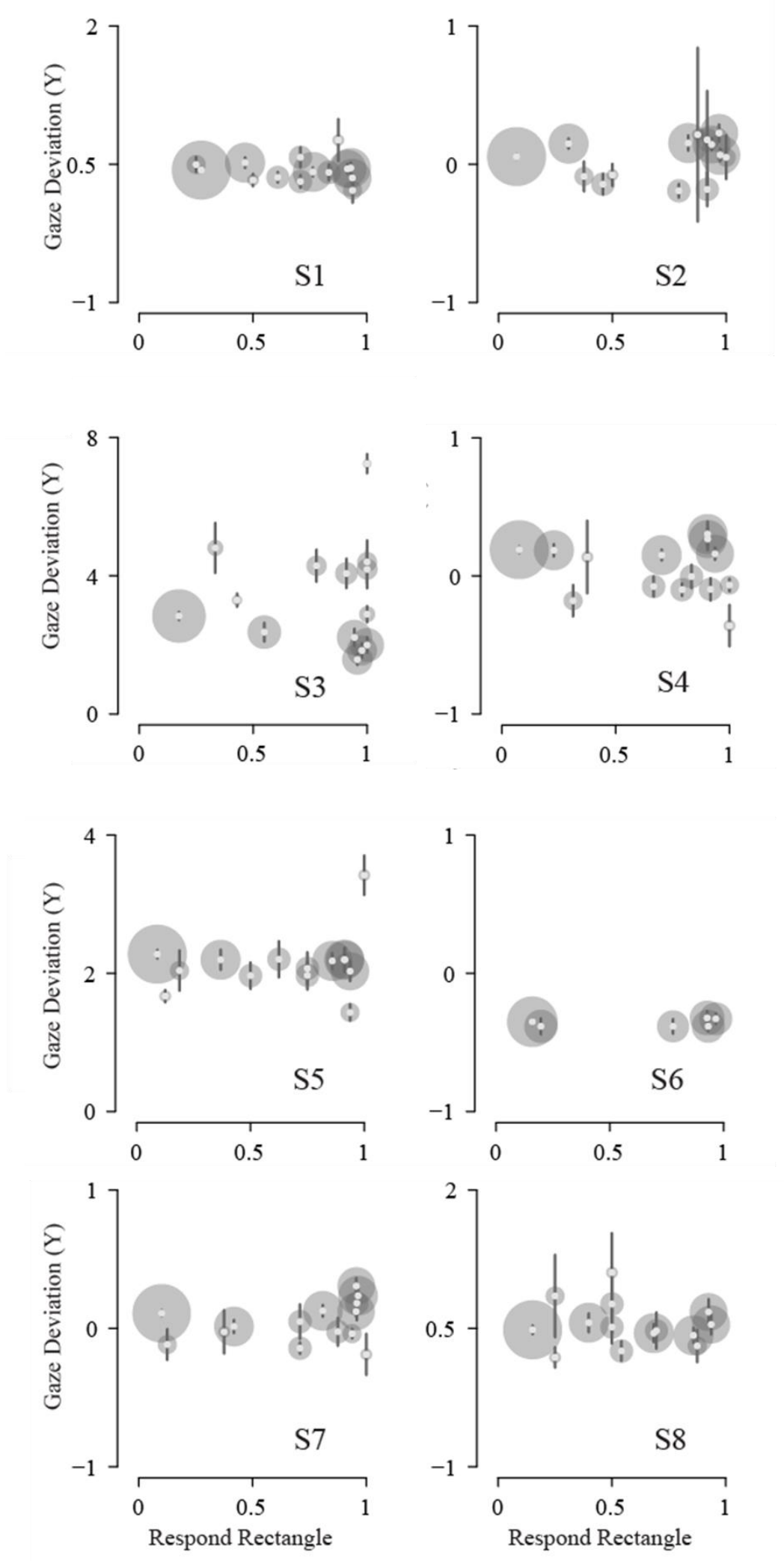

Figure 9. Gaze deviations plotted by proportion of rectangle responses for vertical deviations from the primary horizontal axis of space. Size of circle is proportional to the log of the number of trials. Error bars represent standard errors of the means. 
Given the superiority of fit for the three-parameter Bayesian model, we suggest that the behavioral data reflect both a noisy measurement of length (principal axis major) and width (principal axis minor), and subject-specific decision thresholds. Combining the noisy measurement of PADR with the decision threshold results in a simple binary rule: if the encoded PADR is below the subject's threshold, then report "square"; otherwise report "rectangle." This simple decision rule could also explain the eye-tracking data, in that participants may have attempted to direct the fovea at the centroid of the shape to maximize the number of photoreceptors that fell within the boundaries. Foveating on the centroid of the shape likely allows the extraction of the length and width with maximal sensitivity and recognition accuracy.

We acknowledge that, although the obtained evidence appears more consistent with a principalaxis compared to a medial-axis strategy, eye-tracking data are certainly not definitively and decisively in favor of a principal axis strategy. We suspect that future research may be able to use shapes more complex than those adopted in the present experiment to provide more clear and compelling data to differentiate principal from medial axis strategies (see also Feldman \& Singh, 2006). Regardless, it is quite apparent that eye-tracking data reveal that the underlying strategy adopted by participants is global in nature. In short, eye-tracking data clearly reveal that information about the shape is being extracted from within the shape itself as opposed to comparisons of the lengths of sides. As a result, eye-tracking data appear to rule out any type of strategy based upon the use of local geometric cues such as edge lengths/widths and corner angles and appear consistent with the notion of incidental extraction of global geometric cues (see Cheng, 1986).

Although somewhat limited, the use of eye-tracking technology with nonhuman primates has allowed direct cross-species comparisons of facial processing (Dahl et al., 2009; Kano \& Tomonaga, 2010), and the current paradigm coupled with such technology could be adapted to compare such geometric processing across species. Even in the absence of eye-tracking data, forced choice tasks employing similar geometric stimuli could be utilized to reveal psychophysical functions in a wide variety of species. As importantly, location of contact within the stimuli themselves using touch screen technology may serve as an analog to eye-tracking data.

Collectively, such a comparative approach would appear to have implications for not only shape perception but also orientation research. Take for example, research investigating the role of enclosure size on the relative influence of feature and geometric cues. Research in this area suggests that enclosure geometry exerts greater influence in small enclosures compared to large enclosures, and features exert greater influence in large enclosures compared to small enclosures (Chiandetti, Regolin, Sovrano, \& Vallortigara, 2007; Learmonth, Newcombe, \& Huttenlocher, 2001; Learmonth, Newcombe, Sheridan, \& Jones, 2008; Ratliff \& Newcombe, 2008; Sovrano et al., 2005, Sovrano, Bisazza, \& Vallortigara, 2007; Sturz, Forloines, \& Bodily, 2012; Sturz \& Kelly, 2009; 2013; Vallortigara, Feruglio, \& Sovrano, 2005; see also Miller, 2009). Despite the current lack of systematic psychophysical manipulations of enclosure size to determine difference thresholds, theoretical conclusions have assumed that the size manipulations that have been employed have been sufficient because they have resulted in differences in the relative use of feature and geometric cues. Yet, it seems apparent that a systematic psychophysical manipulation of size is necessary to inform the subjective perception of this factor, which, in turn, is necessary to inform the relative use of geometric and feature cues during size manipulations. Fundamentally, determination of difference thresholds for enclosure size seems critical for accurate and precise predictions regarding the relative use of feature and geometric cues for orientation.

Despite the knowledge that has been gleaned from attempts to determine objective shape parameters by which organisms solve an orientation problem, such an approach seems ill-suited to answer fundamental questions concerning the mechanisms by which organisms determine a heading. We believe that theoretical accounts of spatial orientation can only advance through the integration of knowledge about how participants subjectively perceive and organize space and objective measures of attention (i.e., eye-tracking data). Although limited in scope, our application of this approach to two-dimensional shape perception could be adapted to three-dimensional space. Although the axes in three-dimensional space 
become planes instead of lines, the method of extraction and potential use in shape discrimination could be similar. As importantly, such an approach appears to have relevant and fundamental implications for on-going empirical and theoretical issues regarding how enclosure size and/or shape affect the relative use of feature and geometric cues, as well as highlighting potential similarities and differences in the orientation behavior of individuals. Specifically, such an approach (and the application of Bayesian modeling) has the potential to provide information about biases and noise in spatial decisions, and it seems reasonable to suspect that individuals may well differ with respect to these values. Such knowledge should permit a more diagnostic approach to the delineation of shape parameters and how such information is (or is not) combined with other spatial information (see also Cheng, Shettleworth, Huttenlocher, \& Rieser, 2007). Fundamentally, these psychophysical measurements combined with eyetracking data will not only inform but also advance an ability to determine the mechanisms by which organisms determine a heading.

\section{Author Note}

This research was conducted following the relevant ethical guidelines for human research and supported in part by funds from the Office of the Vice President for Research and the Jack N. Averitt College of Graduate Studies at Georgia Southern University to BRS and TWB.

\section{References}

Ambosta, A. H., Reichert, J. F., \& Kelly, D. M. (2013). Reorienting in virtual 3D environments: Do adult humans use principal axes, media axes or local geometry? PLOS ONE, 8, e78985. doi: 10.1371/journal.pone.0078985

Blum, H. (1967). A transformation for extracting new descriptors of shape. In W. Wathen-Dunn (Ed.), Models for the perception of speech and visual form (pp. 362-380). Cambridge: MA: MIT Press.

Bodily, K. D., Eastman, C. K., \& Sturz, B. R. (2011). Neither by global nor local cues alone: Evidence for a unified orientation process. Animal Cognition, 14, 665-674.

Cheng, K. (1986). A purely geometric module in the rat's spatial representation. Cognition, 23, 149-178.

Cheng, K. (2005). Reflections on geometry and navigation. Connection Science, 17, 5- 21.

Cheng, K. (2012). How to navigate without maps: The power of taxon-like navigation in ants. Comparative Cognition \& Behavior Reviews, 7, 1-22.

Cheng, K., \& Gallistel, C. R. (2005). Shape parameters explain data from spatial transformations: Comment on Pearce et al. (2004) and Tommasi \& Polli (2004). Journal of Experimental Psychology: Animal Behavior Processes, 31, 254-259.

Cheng, K., Huttenlocher, J., \& Newcombe, N. S. (2013). 25 years of research on the use of geometry in spatial reorientation: A current theoretical perspective. Psychonomic Bulletin \& Review, 20, 1033-1054.

Cheng, K., \& Newcombe, N. S. (2005). Is there a geometric module for spatial orientation? Squaring theory and evidence. Psychonomic Bulletin \& Review, 12, 1-23.

Cheng, K., \& Newcombe, N. S. (2006). Geometry, features, and orientation in vertebrate animals: A pictorial review. In M. F. Brown \& R. G. Cook (Eds.), Animal spatial cognition: Comparative, neural, and computational approaches. Retrieved from www.pigeon.psy.tufts.edu/asc/cheng/

Cheng, K., Shettleworth, S. J., Huttenlocher, J., \& Rieser, J. J. (2007). Bayesian integration of spatial information. Psychological Bulletin, 133, 625-637.

Chiandetti, C., Regolin, L., Sovrano, V. A., \& Vallortigara, G. (2007). Spatial reorientation: The effects of space size on the encoding of landmark and geometry information. Animal Cognition, 10, 159-168.

Collett, T. S., \& Zeil, J. (1998). Places and landmarks: An arthropod perspective. In S. Healy (Ed.), Spatial representation in animals (pp. 18-53). Oxford, England: Oxford University Press.

Dahl, C. D., Wallraven, C., Bülthoff, H. H., \& Logothetis, N. K. (2009). Human and macaques employ similar faceprocessing strategies. Current Biology, 19, 509-513.

Feldman, J., \& Singh, M. (2006). Bayesian estimation of the shape skeleton. Proceedings of the National Academy of Sciences, 103, 18014-18019.

Firestone, C., \& Scholl, B. J. (2014). "Please tap the shape, anywhere you like": Shape skeletons in human vision revealed by an exceedingly simple measure. Psychological Science, 25, 377-386. 
Henderson, J. M. (2017). Gaze control as prediction. Trends in Cognitive Sciences, 21, 15-23.

Kano, F., \& Tomonaga, M. (2010). Face scanning in chimpanzees and humans: Continuity and discontinuity. Animal Behaviour, 79, 227-235.

Kardan, O., Berman, M. G., Yourganov, G., Schmidt, J., \& Henderson, J. M. (2015). Classifying mental states from eye movements during scene viewing. Journal of Experimental Psychology: Human Perception and Performance, 41, 1502-1514.

Kelly, D. M., Chiandetti, C., \& Vallortigara, G. (2011a). Re-orienting in space: Do animals use global or local geometry strategies? Biology Letters, 7, 372-375.

Kelly, D. M., \& Durocher, S. (2011). Comparing geometric models for orientation: Medial vs. principal axes. Communicative \& Integrative Biology, 4, 710-712.

Kelly, D. M., Durocher, S., Chiandetti, C., \& Vallortigara, G. (2011b). A misunderstanding of principal and medial axes? Reply to Sturz and Bodily. Biology Letters, 7, 649-650.

Learmonth, A. E., Newcombe, N. S., \& Huttenlocher, J. (2001). Toddlers' use of metric information and landmarks to reorient. Journal of Experimental Child Psychology, 80, 225-244.

Learmonth, A. E., Newcombe, N. S., Sheridan, N., \& Jones, M. (2008). Why size counts: Children's spatial reorientation in large and small enclosures. Developmental Science, 11, 414-426.

Lee, S. A., Sovrano, V. A., \& Spelke, E. S. (2012a). Navigation as a source of geometric knowledge: Young children's use of length, angle, distance, and direction in a reorientation task. Cognition, 123, 144-161.

Lee, S. A., Winkler-Rhoades, N., \& Spelke, E. S. (2012b). Spontaneous reorientation is guided by perceived surface distance, not by image matching or comparison. PLOS ONE, 7, e51373. doi: 10.1371/journal.pone.0051373

Miller, N. Y. (2009). Modeling the effects of enclosure size on geometry learning. Behavioural Processes, 80, 306313.

Pecchia, T., \& Vallortigara, G. (2010). View-based strategy for reorientation by geometry. The Journal of Experimental Biology, 213, 2987-2996.

Ratliff, K. R., \& Newcombe, N. S. (2008). Reorienting when cues conflict: Evidence for an adaptive combination view. Psychological Science, 19, 1301-1307.

R Core Team (2014). R: A language and environment for statistical computing. Vienna, Austria: R Foundation for Statistical Computing.

Sovrano, V. A., Bisazza, A., \& Vallortigara, G. (2007). How fish do geometry in large and in small spaces. Animal Cognition, 10, 47-54.

Sturz, B. R. (2014). Modeling a role of field of view in the extraction of geometric cues during reorientation. Frontiers in Psychology, 5, 535. doi: 10.3389/fpsyg.2014.00535

Sturz, B. R., \& Bodily, K. D. (2011a). Is surface-based orientation influenced by a proportional relationship of shape parameters? Psychonomic Bulletin \& Review, 18, 848-854.

Sturz, B. R., \& Bodily, K. D. (2011b). Of global space or perceived place? Comment on Kelly et al. Biology Letters, 7, 647-648.

Sturz, B. R., \& Bodily, K. D. (2012). On discriminating between geometric strategies of surface-based orientation. Frontiers in Psychology, 3, 112. doi:10.3389/fpsyg.2012.00112

Sturz, B. R., Forloines, M. R., \& Bodily, K. D. (2012). Enclosure size and the use of local and global geometric cues for reorientation. Psychonomic Bulletin \& Review, 19, 270-276.

Sturz, B. R., Gaskin, K. A., \& Roberts, J. E. (2014). Incidental encoding of enclosure geometry does not require visual input: Evidence from blind-folded adults. Memory \& Cognition, 42, 935-942.

Sturz, B. R., Gurley, T., \& Bodily, K. D. (2011). Orientation in trapezoid-shaped enclosures: Implications for theoretical accounts of geometry learning. Journal of Experimental Psychology: Animal Behavior Processes, 37, 246-253.

Sturz, B. R., \& Kelly, D. M. (2009). Encoding of relative enclosure size in a dynamic three-dimensional virtual environment by humans. Behavioural Processes, 82, 223-227.

Sturz, B. R., \& Kelly, D. M. (2013). Environment size and the use of feature and geometric cues for reorientation. Acta Psychologica, 142, 251-258.

Sturz, B. R., Kilday, Z. A., \& Bodily, K. D. (2013). Does constraining field of view prevent extraction of geometric cues for humans during virtual-environment reorientation? Journal of Experimental Psychology: Animal Behavior Processes, 39, 390-396.

Stürzl, W., Cheung, A., Cheng, K., \& Zeil, J. (2008). The information content of panoramic images I: The rotational errors and the similarity of views in rectangular experimental arenas. Journal of Experimental Psychology: Animal Behavior Processes, 34, 1-14. 
Sutton, J. E. (2009). What is geometric information and how do animals use it? Behavioural Processes, 80, 339343.

Vallortigara, G., Feruglio, M., \& Sovrano, V. A. (2005). Reorientation by geometric and landmark information in environments of different size. Developmental Science, 8, 393-401.

Wystrach, A., \& Beugnon, G. (2009). Ants learn geometry and features. Current Biology, 19, 61-66.

Wystrach, A., \& Graham, P. (2012). View-based matching can be more than image matching: The importance of considering an animal's perspective. i-Perception, 3, 547-549. 


\section{Appendix A}

Participant instructions:

In this experiment you will be making perceptual judgments.

First you will see a small cross in the middle of a large circle; fixate the cross.

Next, a shape will appear somewhere within the circle.

Your task is to report whether or not the shape is a square.

If the shape is a square, press "c"/"m".

If the shape is NOT a square, press "m"/"c".

PRESS SPACE TO CONTINUE 